\title{
Development of a Simulation Platform for Underwater Transportation using two Hovering Autonomous Underwater Vehicles (HAUVs)
}

\author{
Faheem Ur Rehman, Giles Thomas, Enrico Anderlini \\ University College London \\ London, United Kingdom \\ ucemfre@ucl.ac.uk; giles.thomas@ucl.ac.uk; e.anderlini@ucl.ac.uk
}

\begin{abstract}
This paper considers two HAUVs undertaking underwater transportation of a spherical payload via cylindrical manipulators. The rigid body connection method of transportation is explored. In this analysis, the nonlinear coupled dynamic model is developed to get an accurate representation of the actual system. Hydrodynamic parameters for all the part bodies are calculated about the centre of the combined system. The hydrostatic terms of the HAUVs and manipulators are selected such that their weight is slightly less than the buoyancy, in order to bring the entire system to surface in case of an emergency. The weight of the payload is selected such that the difference between weight and buoyancy is within the thrust limit of the vertical thrusters on the two HAUVs. The propulsion model is developed taking the effect of all the thrusters on the two HAUVs about the combined centre of body. The simulation platform is developed to observe the response of the entire system and of the individual HAUVs in the system at different revolutions of the thrusters. The stability of the entire system is ensured by maintaining the connection between the vehicles and payload. The simulation results show that stability and motion accuracy are compromised in the axial direction due to the opposite revolutions of the axial thrusters on the two HAUVs.
\end{abstract}

Keywords: Nonlinear coupled dynamic model, Hydrodynamic parameters, Hydrostatic terms, Propulsion model, Stability, Motion accuracy.

\section{Introduction}

Payload transportation is an important requirement for various sectors, which is generally fulfilled by land vehicles, airplanes and sea surface vessels. Subsea transportation can also be used to transport payload; however, it is not commonly used due to the higher density and viscosity of water which increases the hull resistance and power requirement making the vehicle less efficient. Nevertheless, the use of subsea transportation can be necessary in some situations, for example, to avoid the detection of a military payload or to precisely place a subsea installation of an offshore platform etc.

Widespread research has been conducted on transportation using multiple land and aerial vehicles. The three methods deduced are rigid body connection, flexible connection and formation keeping using formation control strategy. In the rigid body connection, the vehicles and the payload are rigidly connected through manipulators. For mathematical modelling, a dynamic model is developed for the entire system considering it a single rigid body [1]. In the flexible connection method, a configuration is established for the vehicles such that the payload is in static equilibrium at the desired position and orientation (pose) keeping in consideration the constraints on cable tension and the payload stability [2]. In the formation keeping approach, the vehicles maintain a formation relative to the payload while transporting it towards the target location. The formation is maintained by means of communication between the vehicles and the payload [3].

The underwater environment is complex compared to land and air, making the implementation of the above mentioned multi-vehicular transportation methods quite challenging. For the land vehicles, the only significant term considered in analysing the motion response is friction, while for the aerial vehicles, gravity is considered the only dominant term [2]. The aerodynamic terms which could have made the analysis quite challenging are ignored due to their less significant values resulting from the small values of density and viscosity of air. On the other hand, underwater vehicles experience some substantial parameters such as the hydrodynamic parameters which consist of the added mass and the damping terms, and the hydrostatic parameters which are influenced by the significant buoyancy effect. Moreover, underwater dynamics is highly nonlinear and coupled. 
The aim is to work out underwater transportation using multiple Unmanned Underwater Vehicles (UUVs). The two main types of UUVs are Remotely Operated Vehicle (ROV) and Autonomous Underwater Vehicle (AUV). ROV is mainly considered a tethered vehicle which is connected via umbilical cable for transferring power and communication [4]. AUV, on the other hand, is an untethered vehicle which contains its own power and controls itself while accomplishing a predefined task [5]. Conventional AUVs are stable at high speed. However, in recent years, AUVs have been developed for the slow operations which are called hovering AUVs (HAUVs) [6]. Moreover, ROVs can also be modified to operate autonomously.

In this work, Minerva ROVs are modified to operate as HAUVs for payload transportation. The rigid body connection method is worked out in which two HAUVs are considered rigidly attached to a spherical payload via cylindrical manipulators.

\section{Dynamic Modelling}

Dynamic modelling is the mathematical representation of the actual system. The model is represented by the equations of motion in six Degrees of Freedom (6 DOF) which include rigid body kinematics and kinetics. It is required to analyse the manoeuvring behaviour as well as designing the control system for an underwater vehicle. This saves the cost of experiments as well as time to evaluate different conditions and situations.

The dynamic model can be developed either nonlinear coupled or linear uncoupled. In this paper, the nonlinear coupled dynamic model is preferred due to its accurate representation of the actual system. However, this issue requires calculation of several parameters as well as complicates the development of dynamic model compared to the linear uncoupled model. The dynamic model is developed based on the approach by Thor I. Fossen [12].

\subsection{Reference Frames}

First, the reference frames are defined i.e. earth-fixed and body-fixed reference frames as shown in Fig. 1. The position and orientation (pose) of the vehicle are represented in the earth-fixed frame, whereas, velocity and forces are defined in the earth-fixed frame. To get the full advantage of the geometric aspects of the body, all the parameters are transformed about the centre of body $(\mathrm{O})$.

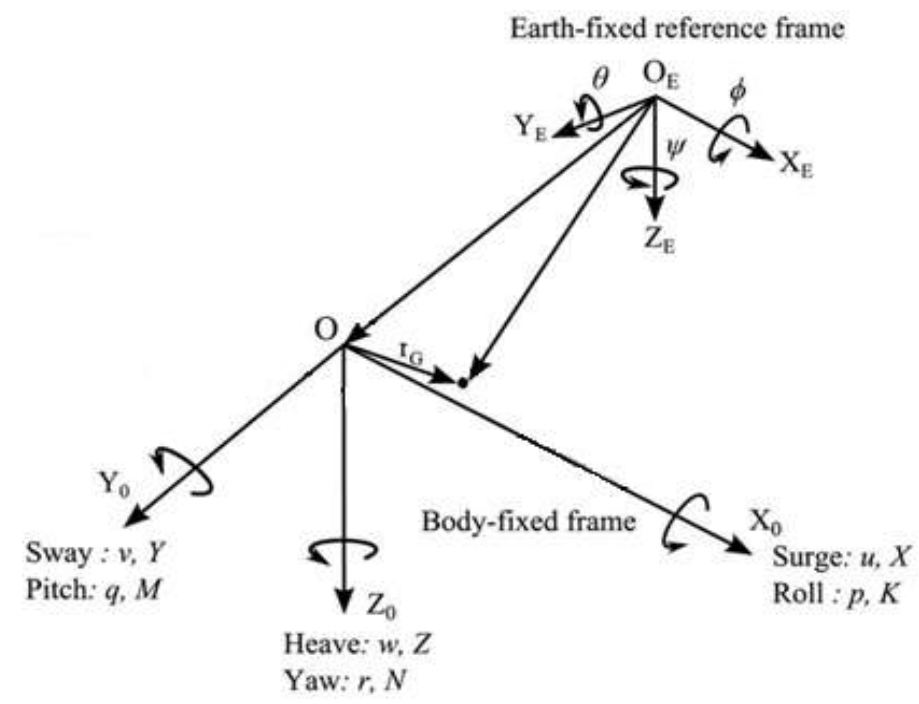

Fig. 1: Frame of References [8].

\subsection{Assumptions}

The development of dynamic model is based on assumptions such as 1) vehicle is a rigid body i.e. the distance between any mass particle on the body and the centre of body does not change by the application of forces [9]. 2) mass and its distribution do not change during the operation of the vehicle. 3) vehicle is deeply submerged so the wave effects are ignored. 4) interaction effects with other bodies and sea current are ignored. Moreover, assumptions are made while calculating the 
hydrodynamic parameters using the empirical data. For instance, DNV standards are used which assumes that the vehicle has 3 planes of symmetry and two of the three sides are equal in dimension or the difference is up to 10\% [10]. These assumptions bring about uncertainties in the dynamic model.

\subsection{Equations of Motion}

Due to the use of same Minerva HAUVs and manipulators, which are attached to the spherical payload in the centre, the combined system has three planes of symmetry. The product of inertia and moment of hydrostatic terms are zero. Moreover, only the diagonal hydrodynamic parameters are considered. Taking the combined centre of body (O) at the combined centre of gravity (COG), the transverse and horizontal components of the combined centre of buoyancy (COB) are zero i.e. $\left[\begin{array}{ll}x_{b} & y_{b}\end{array}\right]=\left[\begin{array}{ll}0 & 0\end{array}\right]$, whereas, the vertical component of COB is above the COG for static stability. The nonlinear coupled equations of motion in 6 DOF of the combined system is written as [7]

$$
\begin{gathered}
\left(m-X_{\dot{u}}\right) \dot{u}+m(w q-v r)-Z_{\dot{w}} w q+Y_{\dot{v}} v r-X_{u} u-X_{u|u|} u|u|+(W-B) \sin \theta=\tau_{X}, \\
\left(m-Y_{\dot{v}}\right) \dot{v}+m(u r-w p)+Z_{\dot{w}} w p-X_{\dot{u}} u r-Y_{v} v-Y_{v|v|} v|v|-(W-B) \cos \theta \sin \phi=\tau_{Y}, \\
\left(m-Z_{\dot{w}}\right) \dot{w}+m(v p-u q)-Y_{\dot{v}} v p+X_{\dot{u}} u q-Z_{w} w-Z_{w|w|} w|w|-(W-B) \cos \theta \cos \phi=\tau_{Z}, \\
\left(I_{x}-K_{\dot{p}}\right) \dot{p}+\left(I_{z}-I_{y}\right) q r+\left(Y_{\dot{v}}-Z_{\dot{w}}\right) v w+\left(M_{\dot{q}}-N_{\dot{r}}\right) q r-K_{p} p-K_{p|p|} p|p|-z_{b} B \cos \theta \sin \phi=\tau_{K}, \\
\left(I_{y}-M_{\dot{q}}\right) \dot{q}+\left(I_{x}-I_{z}\right) r p+\left(Z_{\dot{w}}-X_{\dot{u}}\right) u w+\left(N_{\dot{r}}-K_{\dot{p}}\right) p r-M_{q} q-M_{q|q|} q|q|-z_{b} B \sin \theta=\tau_{M}, \\
\left(I_{z}-N_{\dot{r}}\right) \dot{r}+\left(I_{y}-I_{x}\right) p q+\left(X_{\dot{u}}-Y_{\dot{v}}\right) u v+\left(K_{\dot{p}}-M_{\dot{q}}\right) p q-N_{r} r-N_{r|r|} r|r|=\tau_{N} .
\end{gathered}
$$

Where $m$ is the mass of the entire system which is equal to $m_{1}+2\left(m_{2}+m_{3}\right) . m_{1}, m_{2}$ and $m_{3}$ are the masses of the payload, manipulators and HAUVs respectively. $W$ and $B$ are the weight and buoyancy of the combined system respectively. $I_{x}, I_{y}$ and $I_{z}$ are the moments of inertia of the combined system. $X_{\dot{u}}, Y_{\dot{v}}$ and $Z_{\dot{w}}$ are the translational added mass terms while, $K_{\dot{p}} M_{\dot{q}}$ and $N_{\dot{r}}$ are the rotational added mass terms of the combined system. $X_{u}, Y_{v}, Z_{w}, K_{p}, M_{q}$ and $N_{r}$ are the linear damping terms while, $X_{u|u|}, Y_{v|v|}, Z_{w|w|}, K_{p|p|}, M_{q|q|}$ and $N_{r|r|}$ are the quadratic damping terms of the combined system. $\tau_{X}, \tau_{Y}, \tau_{Z}$, $\tau_{K}, \tau_{M}$ and $\tau_{N}$ are the actuator forces and moments for the combined system.

Note: For the purpose of algorithm generation, these equations were used in matrices.

\subsection{Moments of Inertia}

The moments of inertia of the combined body are shown in equation (1.2). The moments of inertia of HAUVs and manipulators are transformed about $\mathrm{O}$ using the parallel axis theorem.

$$
\begin{gathered}
I_{x}=I_{x 0}+2 I_{x 1}+2 I_{x 2} \\
I_{y}=I_{y 0}+2 I_{y 1}+2 m_{1} x_{\mathrm{b} 1}^{2}+2 I_{y 2}+2 m_{2} x_{b 2}^{2} \\
I_{z}=I_{z 0}+2 I_{z 1}+2 m_{1} x_{\mathrm{b} 1}^{2}+2 I_{z 2}+2 m_{2} x_{b 2}^{2} .
\end{gathered}
$$

$I_{x 1}, I_{y 1}$ and $I_{z 1}$ are the moments of inertia of Minerva HAUV acquired from reference [11]. $x_{b 1}$ and $x_{b 2}$ are the axial distances of the centre of body of HAUV and cylindrical manipulator from O respectively. $m_{1}$ and $m_{2}$ are the masses of HAUV and manipulator respectively. $I_{x 2}, I_{y 2}$ and $I_{z 2}$ are the moments of inertia of the cylindrical manipulator, which are calculated as [12]

$$
\begin{gathered}
I_{x 2}=\frac{1}{12} m a^{2} . \\
I_{y 2}=I_{z 2}=\frac{1}{12} m\left(3 a^{2}+b^{2}\right) .
\end{gathered}
$$

Where $a$ is the radius and $b$ is the length of the cylindrical manipulator. $I_{x 0}, I_{y 0}$ and $I_{z 0}$ are the moments of inertia of the spherical payload which are calculated using the equation [12] 


$$
I_{x 0}=I_{y 0}=I_{z 0}=\frac{2}{5} m a^{2}
$$

Where $a$ is the radius of the spherical payload.

\subsection{Hydrostatics}

The spherical payload is selected of diameter $0.5 \mathrm{~m}$ and having the mass of $130 \mathrm{~kg}$. The difference between the weight and buoyancy of the payload is $630 \mathrm{~N}$. The 4 vertical thrusters on two HAUVs produce a thrust force of $1176 \mathrm{~N}$, therefore, the system will safely lift and move the payload of $130 \mathrm{~kg}$ in the vertical direction. On the other hand, each HAUV is attached to a cylindrical manipulator of length $1 \mathrm{~m}$ and diameter $0.25 \mathrm{~m}$. The weight of the manipulator is $450 \mathrm{~N}$ while the buoyancy is $503 \mathrm{~N}$ which makes the buoyancy of each manipulator slightly higher than its weight.

\subsection{Hydrodynamics}

The hydrodynamic parameters of an underwater vehicle consist of added mass and damping terms. The Morison's formula which is usually used to calculate these parameters is a two-part equation which was derived based on a semiempirical approach [13]. The added mass part was derived based on the potential flow theory and the drag part was based on the experimental analysis. This approach is easier, quicker and provides reasonably accurate results [14].

Hydrodynamic parameters of the Minerva HAUV are acquired from reference [11] and are modified to get their effect about the centre of the combined body. On the other hand, these parameters are calculated for the spherical payload and cylindrical manipulator using Morison's equation [13] where the coefficients are used from references [15][16].

\subsubsection{Hydrodynamic Parameters for the Payload and Manipulator}

For the spherical payload, the translational hydrodynamic parameters are equal in value while the rotational parameters are zero, due to symmetry and the position of centre of payload at O. For the cylindrical manipulator, the translational hydrodynamic parameters in sway and heave are equal in value, the rotational parameter in roll is zero and equal in pitch and yaw, due to symmetry of the body about $\mathrm{x}$-axis.

The added mass part of the Morison's equation for a solid body is given as [15]

$$
A_{i i}=\rho C_{a} V_{R}
$$

$\rho$ is the density of seawater, $C_{a}$ is the added mass coefficient and $V_{R}$ is the reference volume. $C_{a}$ and $V_{R}$ for the spherical payload and cylindrical manipulators are calculated using the empirical data from reference [15].

The damping terms can be linear or nonlinear depending on laminar or turbulent flow respectively. The flow field depends on the Reynolds number ( $\mathrm{Re}$ ) which depends on speed. The speed of multi-vehicular transportation is equal to $1 \mathrm{~m} / \mathrm{sec}$ which is the operational speed of Minerva HAUV. After calculations, it was found that both the spherical payload and cylindrical manipulator lie in the turbulent flow region. Therefore, nonlinear damping terms are dominant. Only the $2^{\text {nd }}$ order nonlinear damping terms are considered because it is difficult to calculate the higher order terms and are also less significant [10].

The quadratic damping or drag from the Morison's equation for a solid body is given as [15]

$$
B_{i i}=\frac{\rho}{2} C_{D} A_{R}
$$

Where $C_{D}$ is the drag coefficient and $A_{R}$ is the reference area. The drag coefficient depends on Re. For the spherical payload, $C_{D}$ is calculated from reference [16] and for the cylindrical manipulator, from reference [15].

After calculation, the hydrodynamic parameters for the spherical payload and cylindrical manipulator are given in Table 1. 
Table 1: Added mass and damping terms for the spherical payload and cylindrical manipulator.

\begin{tabular}{|c|c|c|c|c|c|}
\hline \multicolumn{2}{|c|}{} & $\begin{array}{c}\text { Added mass coefficient } \\
\left(\boldsymbol{C}_{\boldsymbol{a}}\right)\end{array}$ & $\begin{array}{c}\text { Drag coefficient } \\
\left(\boldsymbol{C}_{\boldsymbol{D}}\right)\end{array}$ & $\begin{array}{c}\text { Added } \\
\text { mass terms }\end{array}$ & $\begin{array}{c}\text { Damping } \\
\text { terms }\end{array}$ \\
\hline \multirow{2}{*}{$\begin{array}{c}\text { Spherical } \\
\text { Payload }\end{array}$} & Surge=Sway=Heave & 0.5 & 0.46 & $33.5 \mathrm{~kg}$ & $46.2 \mathrm{~kg} / \mathrm{m}$ \\
\cline { 2 - 6 } & Roll=Pitch=Yaw & - & - & 0 & 0 \\
\hline \multirow{3}{*}{$\begin{array}{c}\text { Cylindrical } \\
\text { Manipulator }\end{array}$} & Surge & $2 / \pi$ & 0.87 & $31.97 \mathrm{~kg}$ & $21.88 \mathrm{~kg} / \mathrm{m}$ \\
\cline { 2 - 6 } & Sway=Heave & 0.852 & 0.65 & $42.8 \mathrm{~kg}$ & $83.28 \mathrm{~kg} / \mathrm{m}$ \\
\cline { 2 - 6 } & Roll & - & - & 0 & 0 \\
\cline { 2 - 6 } & Pitch=Yaw & - & - & $24.1 \mathrm{kgm}^{2}$ & $35.1 \mathrm{kgm}^{2}$ \\
\hline
\end{tabular}

\section{Propulsion Model}

The propulsion model is developed for the 10 thrusters on the two Minerva HAUVs of the combined system. The thrust vector for each thruster about $\mathrm{O}$ is given as [17]

$$
\boldsymbol{\tau}_{\boldsymbol{t}_{\boldsymbol{i}}}=\left[\begin{array}{c}
\boldsymbol{f} \\
\boldsymbol{l}_{\boldsymbol{t}_{\boldsymbol{i}}} \times \boldsymbol{f}
\end{array}\right]=\left[\begin{array}{c}
f_{x} \\
f_{y} \\
f_{z} \\
l_{y} f_{z}-l_{z} f_{y} \\
l_{z} f_{x}-l_{x} f_{z} \\
l_{x} f_{y}-l_{y} f_{x}
\end{array}\right]
$$

Where $\boldsymbol{f}$ is the thrust force vector of the actuator and $\boldsymbol{l}_{\boldsymbol{t}_{\boldsymbol{i}}}$ is the moment arm of the thruster about O.

The thrust vector of the combined system can be written as the product of the thrust allocation matrix $\left(\boldsymbol{T}_{\boldsymbol{a}}\right)$ and thrust force vector $(\boldsymbol{f})$

$$
\tau=T_{a} f
$$

Where, $\boldsymbol{f}$ and $\boldsymbol{T}_{\boldsymbol{a}}$ for the combined system are given in (1. 10) and Error! Reference source not found. respectively.

$$
\boldsymbol{f}=\left[\begin{array}{lllllllllll}
f_{1} & f_{2} & f_{3} & f_{4} & f_{5} & f_{6} & f_{7} & f_{8} & f_{9} & f_{10}
\end{array}\right]^{\boldsymbol{T}} .
$$

Where, $f_{1}$ and $f_{6}$ are the thrust forces of the transverse thrusters, $f_{2} \& f_{3}$ and $f_{7} \& f_{8}$ are the thrust forces of the vertical thrusters and $f_{4} \& f_{5}$ and $f_{9} \& f_{10}$ are the thrust forces of the axial thrusters respectively.

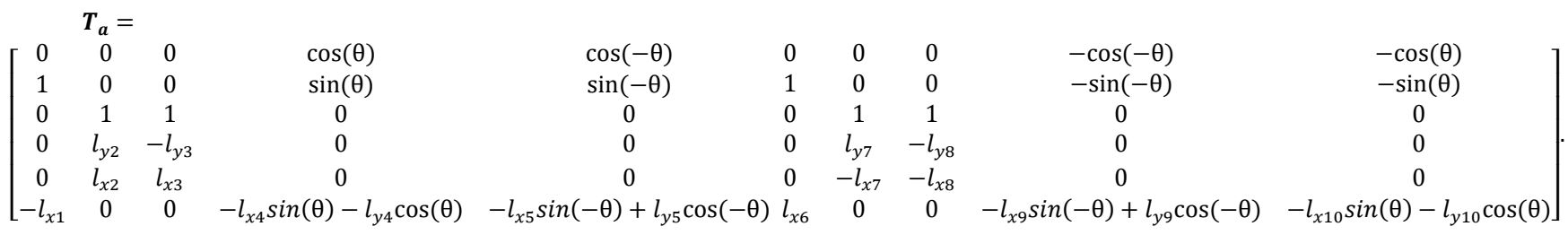

Each column of the thrust allocation matrix represents the contribution of a respective thruster in the combined system about the origin (O). Column 1 and 6 are for the transverse thrusters, column $2 \& 3$ and $7 \& 8$ for the vertical thrusters and column $4 \& 5$ and $9 \& 10$ are for the axial thrusters as shown in Fig. 2. 


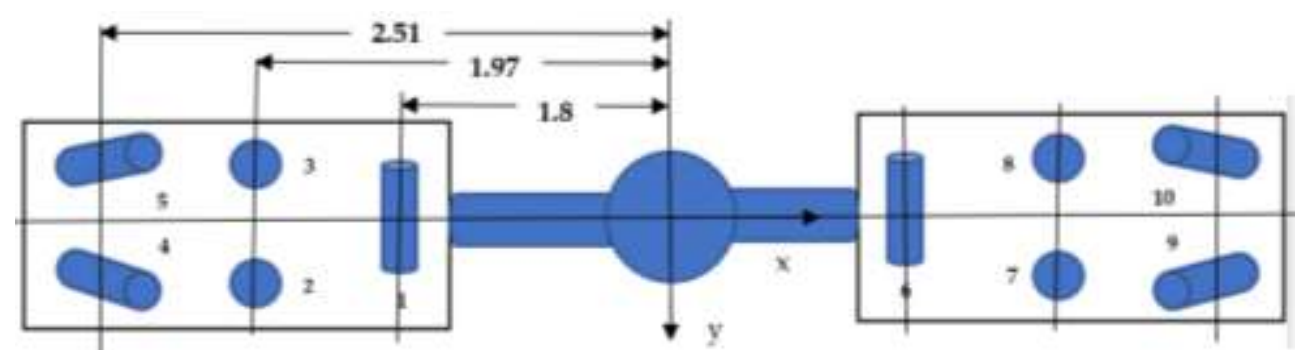

Fig. 2: Allocation of the thrusters in the combined system.

HAUVs approach the payload from the opposite sides, therefore, the axial lever arms are of opposite signs for the two HAUVs. Also, the axial thrusters on the HAUVs act inwards i.e. face each other, therefore, to achieve the axial distance, opposite revolutions are given to the thrusters on the two HAUVs respectively. Furthermore, the axial thrusters are installed at an angle of 10 degrees outward from the axial direction to avoid interaction of flow, therefore, the axial thrust force has components in the $\mathrm{x}$ and $\mathrm{y}$ directions.

The thrust force of a thruster can be calculated by [11]

$$
f=K_{T} \rho D^{4}|n| n .
$$

Where, $D$ is the propeller diameter, $n$ is the revolution per second (rps) and $K_{T}$ is the thrust coefficient. $K_{T}$ can be found from the open water test which gives a relationship between the thrust coefficient $K_{T}$ and the advance ratio of the thruster $J_{a}$. The advance ratio can be calculated as [11]

$$
J_{a}=\frac{V_{a}}{n D} .
$$

$V_{a}$ is called advance velocity which is the velocity of the thruster through water.

The relation between $K_{T}$ and $J_{a}$ for the Minerva HAUV is acquired from [11]. The advance velocity is assumed to be equal to the velocity of the vehicle in the specific direction.

\section{Simulation Platform}

Simulation platform is developed considering the dynamic and propulsion models of the combined system. Running the simulation code provides the motion response of the system over time by giving thruster revolutions as input. The 4th order Runge-Kutta method is used in MATLAB which numerically integrates the system over time with accuracy.

The response of the individual vehicles is also observed in the combined system considering that they are operating independently in the system. In other words, the individual vehicles are assumed attached to the payload but not rigidly fixed to it. Therefore, in case of uncertainties, disturbances or the difference of forces on the two ROVs, they will get apart from the payload. To achieve the response of the individual HAUV in the combined system, separate dynamic models are added in the simulation for each vehicle including manipulator and half the payload.

\subsection{Motion response by applying vertical thrusters}

Fig. 3 shows the motion response when 1000rpm is applied on all the 4 vertical thrusters of the two HAUVs. The vertical distance of $10 \mathrm{~m}$ is covered in 30secs. Each HAUV, if observed independently, maintains the axial distance of $1.97 \mathrm{~m}$ from the payload while moving at the same rate as of the system. 

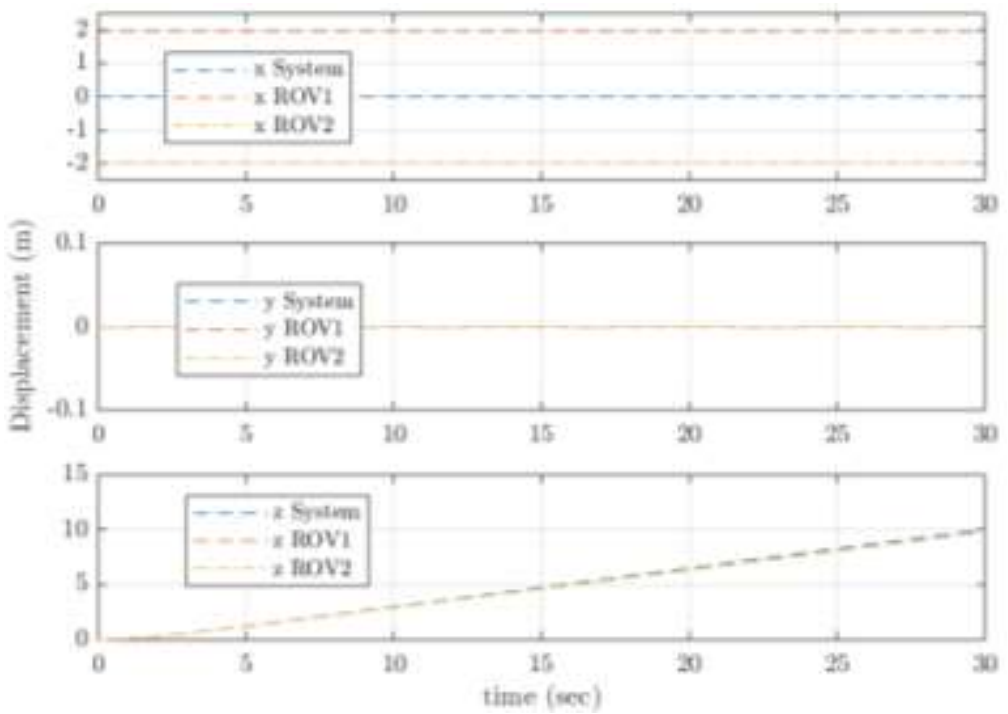

Fig. 3: Motion response for the vertical thrusters.

\subsection{Motion response by applying axial thrusters}

However, when the axial thrusters are applied on the two HAUVs, the vehicles get apart from the payload as time increases. Fig. 4 shows the motion response when 1000rpm is applied on the axial thrusters. The distance between the vehicles and the payload increases and if the vehicles are fixed to the payload, the system starts moving in the sway direction.
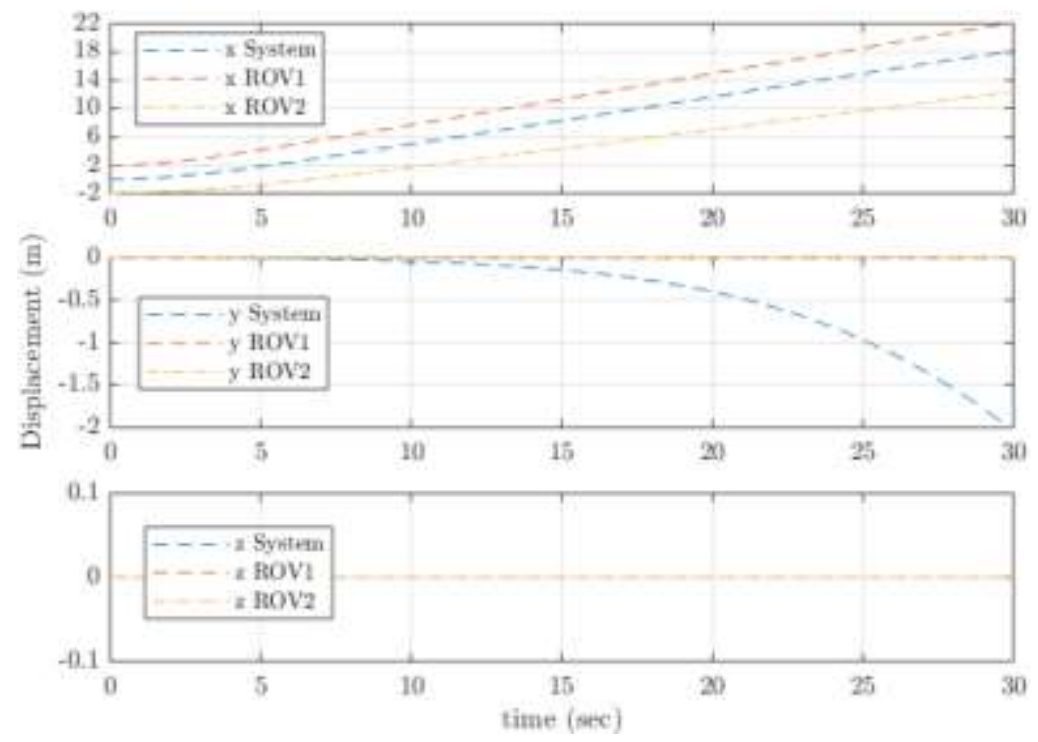

Fig. 4: Motion response for the axial thrusters.

\section{Discussion}

From Section 4.1, it is found that when vertical thrusters are applied, the connection between the HAUVs and payload remains intact as well as the motion response is straight in the vertical direction without any deflection in the other directions. This ensures the stability of the entire system and the desired motion response. However, in Section 4.2, when the axial thrusters are applied either the vehicles get apart or if they are fixed to the payload, the entire system deflect in the sway direction. This means either the payload will be dropped by the vehicles, or the entire system will move in the wrong direction. In both cases, the transportation mission will fail.

The main reason for axial motion inaccuracy or system instability is the opposite revolutions given to the axial thrusters on the two HAUVs of the combined system, in order to move the system in the axial direction. The thrust coefficient $\left(K_{T}\right)$ 
for the Minerva HAUV is greater for the positive revolutions than the negative revolutions [11], therefore, the thrust produced by the axial thrusters of HAUV1 has a higher value than the axial thrusters of HAUV2. Moreover, the axial thrusters are installed at an angle of 10 degrees about the axial direction, therefore, when the HAUVs are bound to the payload, the difference in the transverse thrust forces on the two HAUVs produce a yaw moment which moves the system in the sway direction.

\section{Conclusion}

Stability and accurate motion response are the main requirements for multi-vehicular underwater transportation. The stability is ensured if the vehicles maintain connection with the payload whereas, motion accuracy is ensured if there is no diversion from the desired path during transportation. From the above analysis, it is found that the payload stability and motion accuracy is not ensured in the axial direction due to different thrust forces produced by the axial thrusters on the two HAUVs. There could be other reasons for instability and motion inaccuracy such as disturbances in the underwater environment and hydrodynamic uncertainties. Therefore, a robust control system is required to be designed which is the scope of further work.

\section{References}

[1] D. Mellinger, "Trajectory Generation and Control for Quadrotors," Mechanical Engineering and Applied Mechanics PhD Thesis, University of Pennsylvania, 2012.

[2] N. Michael, J. Fink, and V. Kumar, "Cooperative manipulation and transportation with aerial robots," Auton. Robots, pp. 1-14, 2010.

[3] A. Yufka and M. Ozkan, "Formation-Based Control Scheme for Cooperative Transportation by Multiple Mobile Robots," Int. J. Adv. Robot. Syst., vol. 12, no. 9, 2015.

[4] J. Kennedy, A. A. Proctor, E. Gamroth, and C. Bradley, "Development of a Highly Autonomous Underwater Vehicle for Scientific Data Collection," pp. 1-11.

[5] D. R. Blidberg, "The Development of Autonomous Underwater Vehicles (AUV); A Brief Summary," IEEE ICRA, vol. 6500 , p. 12, 2010.

[6] R. B. Wynn, V. A. I. Huvenne, T. P. Le Bas, B. J. Murton, D. P. Connelly, B. J. Bett, H. A. Ruhl, K. J. Morris, J. Peakall, D. R. Parsons, E. J. Sumner, S. E. Darby, R. M. Dorrell, J. E. Hunt, "Autonomous Underwater Vehicles (AUVs): Their past, present and future contributions to the advancement of marine geoscience," Mar. Geol., 2014.

[7] T. I. Fossen, HandBook of Marine Craft Hydrodynamics and Motion Control, 1st Ed. Sussex: John Wiley \& sons, 2011.

[8] P. S. Londhe, B. M. Patre, L. M. Waghmare, and M. Santhakumar, "Robust proportional derivative (PD)-like fuzzy control designs for diving and steering planes control of an autonomous underwater vehicle," J. Intell. Fuzzy Syst., vol. 32, no. 3, pp. 2509-2522, 2017.

[9] J. Peraire and J. Widnall, "Lecture L26 - 3D Rigid Body Dynamics : The Inertia Tensor," in Dynamics, 2008.

[10] O. A. Eidsvik, "Identification of Hydrodynamic Parameters for Remotely Operated Vehicles," Marine Technology Master Thesis, Norwegian University of Science and Tehnology, 2015.

[11] S. Marie Mo, "Development of a Simulation Platform for ROV systems," Marine Technology Master Thesis, Norwegian University of Science and Technology, 2015.

[12] "Moment of inertia equation ad formulas of rigid objects," Physics about, 2018. [Online]. Available: https://physicsabout.com/moment-of-inertia/.

[13] J. R. Morison, M. P. O'Brien, J. W. Johnson, and S. A. Schaf, "The force exerted by surface waves on piles," Pet. Technol., vol. 2, no. 5, 1950.

[14] A. D. Madan and M. T. Issac, "Hydrodynamic Analysis of AUV Hulls Using Semi-empirical and CFD Approach," Univers. J. Mech. Eng., vol. 5, no. 5, pp. 137-143, 2017.

[15] DNV, "Modelling and Analysis of Marine Operations," tech. rep. DNV-RP-H103, pp. 1-91, 2010.

[16] M. D. Mikhailov and A. P. S. Freire, "The drag coefficient of a sphere: An approximation using Shanks transform," Powder Technol., vol. 237, pp. 432-435, 2013.

[17] S. S. Sandøy, "System Identification and State Estimation for ROV uDrone," Marine Technology Master Thesis, Norwegian University of Science and Technology, 2016. 